\title{
Determination of physical parameters of five large amplitude $\delta$ Scuti stars
}

\author{
J.H. Peña, D. González, and R. Peniche \\ Instituto de Astronomía, UNAM, Mexico Apartado Postal 70-264, D.F., Mexico
}

Received December 30, 1997; accepted March 1, 1999

\begin{abstract}
. $u v b y-\beta$ photometry of the large amplitude $\delta$ Scuti stars BP Peg, DY Peg, DY Her, CY Aqr and YZ Boo is presented. Since the data were obtained almost simultaneously in all filters, meaningful physical parameters have been derived for each star along their pulsation cycles using the calibrations from Nissen (1988) for $A$ and $F$ stars to determine the reddening and derive the unreddened photometric values. The utilization of the theoretical grids of Relyea \& Kurucz (1978) has allowed temperature and gravity values to be determined. A comparison with previously reported values is presented.
\end{abstract}

Key words: stars: fundamental parameters $-\delta$ Sct

\section{Introduction}

The determination of physical parameters such as effective temperature and superficial gravity can be done through the Strömgren photometric data reduced to the standard system for $B-F$ stars, once corrected for interstellar extinction. This procedure has been done previously by the authors for $\delta$ scuti variables and for stars in open cluster which lie within these spectral; its description can be found in Peniche et al. (1990), for example.

We must emphasize that for these fast pulsators, some of which are multi-periodic or with unexplained light curve variations, the data gathered in the present work has the advantage that it need not be adjusted in phase as it would have been with a one-channel photometer. This also allows an extensive time coverage along the cycle, in particular if the star is faint. On the other hand, the simultaneous measurements in the different filters that define the Strömgren system let us avoid problems due to interpolation which worsen if more than one frequency is present since it is well known (Breger 1998) that many high amplitude $\delta$ Scuti stars (HADS) are double mode radial pulsators. Hence,

Send offprint requests to: J.H. Peña the analysis of the photometry presented in the current work shows the real variation of the physical characteristics of the stars along their pulsation cycles.

If the photometric system is well-defined and calibrated, it will provide an efficient way to investigate physical conditions. A comparison with theoretical models, such as those of Relyea \& Kurucz (1978), allows a direct comparison with intermediate or wide band photometry measured from the stars with those obtained theoretically for early type stars. Relyea \& Kurucz (1978) calculated grids for stellar atmospheres for G, F, A, B and O stars for the solar abundance $[\mathrm{Fe} / \mathrm{H}]=0.00$ in a temperature range from $5500 \mathrm{~K}$ up to $50000 \mathrm{~K}$. They also considered abundances of 0.1 and 0.001 . A comparison of the photometric unreddened indexes $(b-y)_{0}$ and $c_{0}$ obtained for each star with such models permits the determination of the effective temperature Te and superficial gravity $\log g$ along the cycle of pulsation. Therefore, the research goals for $\delta$ Scuti stars should be to try to determine not only the frequency content but also the maximum number of physical characteristics of as many stars as possible, along each star's cycle variation. Furthermore, there still exists the question of the real physical differences between the $\delta$ Scuti stars and Dwarf Cepheids; Breger (1980) stated that the majority of dwarf Cepheids mimic Pop I $\delta$ Scuti stars in basically all aspects, except for a few stars in the subgroup of Dwarf Cepheids known as the SX Phe that show low metallicity, high space velocities and low luminosities and that do not conform the theoretical period gravity relation in the direction of low mass. Hence motivation of the present work.

\section{Observations}

The photometric system utilized has the advantage, that the uvby photometry is acquired simultaneously and the $N$ and $W$ filters that define $\mathrm{H} \beta$ almost simultaneously. All the observations were carried out at the Observatorio Astronómico Nacional at San Pedro Mártir, Mexico. 
Table 1. Uncertainties in the color indexes $V,(b-y), m_{1}, c_{1}$ and $\beta$

\begin{tabular}{lrrrrrr}
\hline Season & star & $\delta V$ & $\delta(b-y)$ & $\delta m_{1}$ & $\delta c_{1}$ & $\delta \beta$ \\
\hline 1986A & BP Peg & 0.017 & 0.011 & 0.011 & 0.013 & 0.009 \\
1986B & DY Her & 0.009 & 0.008 & 0.007 & 0.011 & 0.005 \\
1992A & DY Peg & 0.023 & 0.013 & 0.024 & 0.039 & 0.022 \\
$1992 B$ & CY Aqr & 0.021 & 0.008 & 0.007 & 0.010 & 0.007 \\
1994 & YZ Boo & 0.015 & 0.013 & 0.017 & 0.018 & 0.005 \\
\hline
\end{tabular}

The data was acquired in different observing seasons: July-August, 1986; August-September, 1986; SeptemberOctober, 1992 and March, 1994. All were carried out at the $1.5 \mathrm{~m}$ telescope with the same spectrophotometer. A description of this instrument can be found in Nissen (1984). The reduction procedure was done through the numerical packages NABAPHOT (Arellano-Ferro \& Parrao 1989) and DAMADAP (Parrao et al. 1998) which reduce the data into a standard system. The system that was chosen was that defined by the standard values of Olsen (1983) and the transformation equations are those defined by Crawford \& Barnes (1970) and by Crawford \& Mander (1966). An estimate of accuracy was done by comparing the $u v b y-\beta$ data obtained with those of Olsen (1983) and Grönbech \& Olsen (1977). The uncertainties were evaluated in the following manner: The average differences, present data of the standard stars minus Olsen's data, were evaluated and provide an uncertainty for the transformation of the season, see Table 1 . These values, of course, are functions of the star magnitude and in all cases enough star counts were secured in order to attain a signal to noise ratio large enough to determine an accuracy better than $0.01 \mathrm{mag}$, small compared to the large amplitude variation of these stars. The final photometric values have been submitted to the IAU archive but the mean Strömgren indices which cover a whole cycle and the range of the variation due to pulsation for each star are listed in Table 2.

\section{Discussion}

In order to carry out the previously described techniques, the photometric values in the standard system presented in the section of Observations were used first to determine reddening. This was done using specific techniques which have been previously described (see, for example, Peña et al. 1993) and which are based on a calibration by Nissen (1988) based, in turn, on the fundamental calibrations of Crawford and his co-workers $(1975,1979)$ for A and F stars. This is applicable since, as is well-known, the $\delta$ Scuti stars are within these spectral limits in the HR diagram. For both types, Crawford derived empirical calibrations for $M_{v}$ and $(b-y)_{0}$ and in the latter case, also the $[\mathrm{Fe} / \mathrm{H}]$ metallicity. With these calibrations, slightly modified by Nissen (1988), a computing code was written to determine reddening and unreddened parameters for each set of photometric data. A slight interpolation would be necessary since the $\mathrm{H} \beta$ values differ slightly in time from uvby photometry. However, since the difference is so small, in practice they were treated as if they were taken at the same time.

A brief description for each star is given which emphasizes the main characteristics relevant to the findings of the present paper:

BP Peg. Broglia (1959) reported a linear ephemeris with a period of $0.10954347 \mathrm{~d}$, detecting also a possible variation of the period between the 1953 and 1958 and 1959 observations. Furthermore, they found a modulation period on the order of $0.37 \mathrm{~d}$ with an amplitude in the maxima of $0.45 \mathrm{mag}$ in the visual. Rodríguez (1989) presented uvby observations of this star and identified two main frequencies, 9.1260 and $11.8303 \mathrm{c} / \mathrm{d}$ corresponding to the fundamental mode and to the first overtone. Kim et al. (1989) secured new $u v b y-\beta$ and spectroscopic observations of this star. They determined a photometric reddening value of $E(b-y)$ equal to 0.067 mag. A superficial gravity value of 3.85 and a ratio $[\mathrm{Fe} / \mathrm{H}]$ of -0.08 were obtained. An average $T_{\mathrm{e}}$ of $7470 \mathrm{~K}$ was determined from their photometry and theoretical grids. From this and theoretical models, a bolometric magnitude of $M_{\mathrm{bol}} 1.4 \mathrm{mag}$, a mass of $1.85 M_{\odot}$ and an age of $1.310^{9} \mathrm{yr}$ were finally derived. Rodríguez et al. (1992) also reported uvby- $\beta$ of three large amplitude $\delta$ Scuti stars, BP Peg, among them. They were able to determine the same parameters previously established with their photometry.

CY Aqr. Detre \& Chang (1960) and Hardie \& Tolbert (1961) reported an ephemeris with a negative quadratic term. Percy (1975) reported that the period remained constant between 1934 and 1951, when it suffered a drastic change and then remained constant. Elst (1972) determined a modulation period of $0.1222 \mathrm{~d}$, but with a very small amplitude. Fitch (1973) suggested the modulation period might be $0.17766 \mathrm{~d}$, which could be explained by the pulsational theory, whereas Elst's (1972) period, could not. Percy (1975) found that the modulation period proposed by Elst (1972) was not real but merely a result of its method of analysis. Geyer \& Hoffmann $(1974,1975)$ did not find any modulation period. Mahdy \& Szeidl (1980) derived an abrupt change of the period of $-18.110^{-8} \mathrm{~d}$ around 1952 , the period being constant before and after this date. Rolland et al. (1986) and Peña et al. (1987), proposed a period decay consistent with the theoretical model of a pre-white dwarf of $0.2 M_{\odot}$ in opposition to the abrupt period changes suggested. Rodríguez (1989) determined a linear ephemeris for his data and, in a later study, (Rodríguez et al. 1990) determined some observational parameters such as effective temperature of Te $7590 \mathrm{~K}$, superficial gravity of $\log g=3.89$ and metallicity $[\mathrm{Fe} / \mathrm{H}]=-0.7$. Besides these values, they determined, considering pulsation, the following: $R_{*}=2.34 R_{\odot}, M=1.49 M_{\odot}, M_{\mathrm{bol}}=1.71$ and an 
Table 2. Mean Strömgren indices and the range of the variation for each star

\begin{tabular}{lccccc}
\hline & BP Peg & CY Aqr & DY Her & DY Peg & YZ Boo \\
\hline$\langle V\rangle$ & 11.9628 & 10.7459 & 10.5797 & 10.3688 & 10.5849 \\
$V_{\max }$ & 11.68 & 10.421 & 10.402 & 10.057 & 10.311 \\
$V_{\min }$ & 12.17 & 11.096 & 10.803 & 10.613 & 10.730 \\
$\langle b-y\rangle$ & 0.2165 & 0.1844 & 0.2091 & 0.1738 & 0.1688 \\
$(b-y)_{\max }$ & 0.154 & 0.115 & 0.137 & 0.104 & 0.113 \\
$(b-y)_{\min }$ & 0.274 & 0.272 & 0.276 & 0.229 & 0.189 \\
$\left\langle m_{1}\right\rangle$ & 0.2244 & 0.1165 & 0.2061 & 0.1444 & 0.1458 \\
$m_{1} \max$ & 0.267 & 0.152 & 0.172 & 0.176 & 0.160 \\
$m_{1} \min$ & 0.173 & 0.066 & 0.242 & 0.118 & 0.134 \\
$\left\langle c_{1}\right\rangle$ & 0.7399 & 0.8937 & 0.7839 & 0.9125 & 0.8374 \\
$c_{1} \max$ & 0.861 & 1.045 & 0.928 & 1.069 & 0.956 \\
$c_{1} \min$ & 0.628 & 0.718 & 0.642 & 0.772 & 0.771 \\
$\langle\beta\rangle$ & 2.7636 & 2.7994 & 2.7503 & 2.7879 & 2.7520 \\
$\beta_{\max }$ & 2.853 & 2.877 & 2.811 & 2.857 & 2.810 \\
$\beta_{\min }$ & 2.690 & 2.697 & 2.700 & 2.708 & 2.715 \\
\hline
\end{tabular}

age of $1.710^{9}$ yr. Coates et al. (1994) determined a period change and concluded that the star was pulsating with only one period. This conclusion is not supported by the study of Powell et al. (1995) which stated that the new data did not present significant variations from cycle to cycle, but that an abrupt period change occurred in 1989 and that the star is now pulsating with a period of $0.061038612 \mathrm{~d}$.

DY Her. Since its discovery as a variable star in the thirties it has been a subject of considerable interest. In works by McNamara (1978) with spectra and by Breger et al. (1978) from $u v b y-\beta$, a value of $2.7 R_{\odot}$ was determined. Szeidl (1979) stated that the radius should be larger than that previously reported. Breger et al. (1978) determined the effective temperature and superficial gravity with Strömgren photometry with a one channel photometer. Rodríguez (1989) determined the metallicity of the star with simultaneous Strömgren photometry. López de Coca et al. (1990) included it in a comprehensive review of the observational characteristics of all the $\delta$ Scuti stars known. Antonello (1990) carried out a comparison with theoretical models concerning the Strömgren $m_{1}$ index. For DY Her he derived $\left\langle T_{\mathrm{e}}\right\rangle$ of $7360 \mathrm{~K}, \log \langle g\rangle$ of 3.87 and $[\mathrm{Fe} / \mathrm{H}]$ of 0.16 . The physical parameters of mass, bolometric magnitude and age were later determined from uvby $-\beta$ photometry by Rodríguez et al. (1990). Dawei et al. (1993) confirmed the period change of DY Her based on studies by Madhy \& Szeidl (1980) with a compilation of 60 maxima from 1938 up to 1979. Yang et al. (1993) made a compilation of 30 years of observations determining a variation in the period of $P_{0}=0.14863130 \mathrm{~d}$, $1 / p(\mathrm{~d} p / \mathrm{d} t)=-5.210^{-9} \mathrm{yr}$. Milone et al. (1994) derived the mean radius and bolometric luminosity of $2.77 R_{\odot}$ and $M_{\text {bol }}=1.41 \mathrm{mag}$, respectively, from visual and infrared photometry and the effective temperature derived by Breger et al. (1978).

DY Peg. An excellent review of the previous observations can be found in Rodriguez (1989). Between 1966 and 1975 several observers obtained new photometric data with the purpose of confirming light curve variations. Quigley \& Africano $(1979,1981)$ derived a quadratic ephemeris which predicted a decrease in the period. Peña $\&$ Peniche (1986) derived a decrease in the period, consistent with the theoretical models of Dziembowski \& Kozlowski (1974) for a pre-white-dwarf in opposition to the abrupt period changes proposed by Percy (1975) and Mahdy \& Szeidl (1980). Rodriguez (1989) reported observations of DY Peg in the Strömgren system determining a period of 0.0729 d. Recently, Kilambi \& Rahman (1993) presented $U B V$ observations and determined mass, radius, gravity and variations of the temperature over one cycle of pulsation. Rodriguez et al. (1993) reported light curves in the $u v b y-\beta$ system.

YZ Boo. Studies by Szeidl \& Mahdy (1981) led them to conclude that YZ Boo had suffered a period change. Joner \& McNamara (1983) derived its physical parameters $\left(T_{\mathrm{e}}=7590\right.$ and $\log g=3.95,[\mathrm{Fe} / \mathrm{H}]=-0.025$, $\left.M_{\mathrm{bol}}=+1.7\right)$ through $U B V$ and Strömgren photometry and concluded that it is a dwarf Cepheid of Pop I, which, according to Breger (1979) could be considered to be $\delta$ Scuti star. Since the period changes can provide information on the evolutive stage of the stars, several studies have been done with this goal in mind: this star was first studied by Szeidl \& Mahdy (1981), later by Peniche et al. (1985); Jiang (1986), determined new ephemeris with a quadratic term. Peniche et al. (1985) concluded that it is impossible to decide if the period is constant or not with the currently available data bases. Kim \& Joner (1994a) reported photometric observations in Coussins VRc system and in a later paper Kim \& Joner (1994b) determined $T_{e}=7410 \mathrm{~K}, \log g=3.71$ and a metallicity $[M / H]=-0.025$.

\section{Analysis}

Simultaneous $u v b y-\beta$ photoelectric photometry for the $\delta$ Scuti stars CY Aqr, DY Peg, YZ Boo, BP Peg, and DY Her has been secured. The application of the previously 
Table 3. Unreddened photometry of BP Peg

\begin{tabular}{|c|c|c|c|c|c|c|c|}
\hline Time & $(b-y)_{0}$ & $m_{10}$ & $c_{10}$ & Time & $(b-y)_{0}$ & $m_{10}$ & $c_{10}$ \\
\hline 3.7745 & 0.144 & 0.217 & 0.836 & 3.8431 & 0.222 & 0.201 & 0.652 \\
\hline 3.7777 & 0.140 & .239 & 0.832 & 3.8474 & & 0.264 & 0.628 \\
\hline 3.7789 & 0.127 & 0.263 & 0.810 & 3.8489 & 0.203 & 0.236 & 0.619 \\
\hline 3.7877 & 0.173 & 0.199 & 0.797 & 3.8503 & 0.218 & 0.207 & 0.636 \\
\hline 3.7890 & 0.161 & 0.208 & 0.789 & 3.8519 & 0.200 & 0.243 & 0.618 \\
\hline 3.7902 & 0.163 & .216 & 0.795 & 3.8556 & 0.197 & 0.232 & 0.630 \\
\hline 3.7929 & 0.161 & .239 & 0.760 & 3.8569 & 0.192 & 0.237 & 0.657 \\
\hline 3.7941 & 0.173 & 0.207 & 0.780 & 3.8581 & 0.202 & 0.220 & 0.652 \\
\hline 3.7956 & 0.180 & 0.214 & 0.750 & 3.8609 & 0.175 & 0.255 & 0.636 \\
\hline 3.7969 & & & 0.7 & & 85 & 39 & 0.658 \\
\hline 3.8000 & 0.189 & .191 & 0.751 & 3.86 & 0.187 & 0.221 & 0.669 \\
\hline 3.8013 & 0.173 & .224 & 0.767 & & 0.162 & 0.257 & 0.658 \\
\hline 3.8045 & 0.206 & 0.180 & 0.738 & 3.86 & 57 & 0.257 & 0.700 \\
\hline 3.8058 & 0.198 & 0.193 & 0.748 & 3.86 & 0.146 & 0.255 & 0.714 \\
\hline 3.8070 & 182 & .229 & 0.710 & 3.86 & 0.134 & 0.252 & 0.774 \\
\hline 3.8100 & 0.175 & 0.254 & 0.688 & 3.8711 & 0.138 & 0.245 & 0.757 \\
\hline 3.8113 & 0.173 & 0.253 & 0.705 & 3.8723 & 0.118 & 0.265 & 0.778 \\
\hline 3.8126 & 0.180 & 0.239 & 0.696 & 3.8737 & .123 & 0.251 & 0.796 \\
\hline 3.8138 & 0.200 & 0.205 & 0.706 & 3.8750 & 0.108 & 0.275 & 0.803 \\
\hline 3.8166 & 0.200 & 0.212 & 0.688 & 3.8777 & 0.108 & 0.256 & 0.832 \\
\hline 3.8180 & 0.206 & .204 & 0.687 & 3.8790 & .113 & 0.250 & 0.838 \\
\hline 3.8193 & 0.202 & 0.208 & 0.686 & 3.8803 & 0.110 & 0.253 & 0.845 \\
\hline 3.8205 & 0.203 & 0.211 & 0.691 & 3.8817 & 0.112 & 0.251 & 0.844 \\
\hline 3.8236 & 0.213 & 0.188 & 0.720 & 3.8857 & 0.113 & 0.252 & 0.852 \\
\hline 3.8250 & & & 0.677 & & & & 0.835 \\
\hline 3.8262 & 0.210 & 0.192 & 0.705 & 3.8901 & 0.123 & 0.275 & 0.800 \\
\hline 3.8285 & 0.200 & 0.228 & 0.670 & 3.8915 & 0.117 & 0.267 & 0.818 \\
\hline 3.8297 & 0.205 & 0.221 & 0.674 & 3.8927 & 0.140 & 0.229 & 0.826 \\
\hline 3.8310 & 0.208 & 0.214 & 0.665 & 3.8939 & 0.130 & 0.256 & 0.816 \\
\hline 3.8322 & 0.228 & 0.195 & 0.668 & 3.8953 & 0.136 & 0.257 & 0.787 \\
\hline 3.8335 & 0.198 & 0.232 & 0.672 & 3.8983 & 0.148 & 0.242 & 0.792 \\
\hline 3.8365 & 0.195 & 0.237 & 0.660 & 3.9000 & 0.154 & 0.237 & 0.789 \\
\hline 3.8378 & 0.204 & 0.228 & 0.653 & 3.9013 & 0.153 & 0.243 & 0.768 \\
\hline 3.8390 & 0.208 & 0.222 & 0.652 & 3.9027 & 0.161 & 0.238 & 0.778 \\
\hline 3.8404 & & 0.264 & 0.640 & 3.9056 & & 0.248 & 0.752 \\
\hline 3.8419 & 0.207 & 0.214 & 0.646 & 3.9069 & 0.168 & 0.245 & 0.744 \\
\hline
\end{tabular}

$\mathrm{HJD}=$ time +2446670.0 .

described technique from Peña et al. (1993) to the data for each star provides the reddening for each set of colors obtained at the same time. A mean value of the reddening was considered and from this, unreddened indexes were obtained. Once the unreddened indexes have been evaluated, (Tables 3 to 7 for BP Peg, CY Aqr, DY Her, DY Peg and YZ Boo, respectively) the location of each data point was determined in the theoretical grids of Relyea \& Kurucz (1978) for solar abundance and lower. From the photometry, superficial gravity and effective temperatures as well as metallicity, absolute magnitude and distance have been derived for each star and the variations in $T_{\mathrm{e}}$ and $\log g$ during the pulsation cycle for the large amplitude stars is presented in Table 8. A compilation of the values reported in the literature has been done and the derived values compared well with those determined previously (Table 9). In particular CY Aqr and DY Peg show low metallicity, a characteristic of Pop II stars whereas DY Her and YZ Boo have Pop I metallicity.

Since the values of metallicity found for BP Peg differ from those previously compiled, a careful analysis was carried out: a direct comparison with another source of uvby - $\beta$ (KMJ, 1989) was done. Since significant values for determining physical quantities are those between phases 0.3 to 0.75 (Breger et al. 1978; McNamara 1997), phase diagrams were constructed with the KMJ photometry of the longest nights $(2447413 \& 20)$ to avoid the beating phenomena that could arise from the two determined frequencies in BP Peg. What was immediately evident, was that most of the measured $\mathrm{H} \beta$ values for $\mathrm{BP}$ Peg from the two sources were above the 2.72 limit that establishes the difference between $\mathrm{A}$ and $\mathrm{F}$ type stars since $[\mathrm{Fe} / \mathrm{H}]$ can 
Table 4. Unreddened photometry of CY Aqr

\begin{tabular}{lccccccc}
\hline Time & $(b-y)_{0}$ & $m_{10}$ & $c_{10}$ & Time & $(b-y)_{0}$ & $m_{10}$ & $c_{10}$ \\
\hline 0.632 & 0.148 & 0.1288 & 0.7712 & 0.6584 & 0.171 & 0.1208 & 0.8142 \\
0.6346 & 0.111 & 0.1438 & 0.8842 & 0.6632 & 0.194 & 0.0978 & 0.7832 \\
0.6356 & 0.083 & 0.1708 & 0.9062 & 0.6644 & 0.199 & 0.0788 & 0.8022 \\
0.6365 & 0.04 & 0.1538 & 1.0012 & 0.6773 & 0.21 & 0.1028 & 0.7082 \\
0.6379 & 0.069 & 0.1598 & 1.0042 & 0.6798 & 0.224 & 0.0818 & 0.7202 \\
0.6401 & 0.077 & 0.1518 & 1.0182 & 0.6815 & 0.215 & 0.0878 & 0.7162 \\
0.6412 & 0.071 & 0.1518 & 1.0332 & 0.6829 & 0.215 & 0.0888 & 0.7152 \\
0.6431 & 0.086 & 0.1398 & 1.0322 & 0.6857 & 0.217 & 0.0748 & 0.7272 \\
0.6456 & 0.098 & 0.1428 & 1.0022 & 0.6928 & 0.171 & 0.1008 & 0.7872 \\
0.6467 & 0.102 & 0.1598 & 0.9732 & 0.6941 & 0.145 & 0.1258 & 0.8072 \\
0.6485 & 0.128 & 0.1278 & 0.9622 & 0.695 & 0.126 & 0.1378 & 0.8482 \\
0.6494 & 0.122 & 0.1398 & 0.9232 & 0.696 & 0.113 & 0.1288 & 0.9092 \\
0.6512 & 0.142 & 0.1388 & 0.8732 & 0.6982 & 0.081 & 0.1378 & 0.9872 \\
0.6526 & 0.153 & 0.1128 & 0.9122 & 0.7001 & 0.071 & 0.1398 & 1.0322 \\
0.6545 & 0.161 & 0.1258 & 0.8522 & 0.7017 & 0.084 & 0.1328 & 1.0322 \\
0.6561 & 0.17 & 0.1008 & 0.8622 & & & & \\
\hline
\end{tabular}

HJD $=$ time +2444494.0.

Table 5. Unreddened photometry of DY Her

\begin{tabular}{lccccccc}
\hline Time & $(b-y)_{0}$ & $m_{10}$ & $c_{10}$ & Time & $(b-y)_{0}$ & $m_{10}$ & $c_{10}$ \\
\hline 0.6653 & 0.127 & 0.197 & 0.946 & 0.7594 & 0.250 & 0.204 & 0.649 \\
0.6697 & 0.137 & 0.191 & 0.951 & 0.7633 & 0.252 & 0.210 & 0.637 \\
0.6743 & 0.139 & 0.192 & 0.933 & 0.7722 & 0.245 & 0.215 & 0.632 \\
0.6787 & 0.150 & 0.188 & 0.921 & 0.7762 & 0.249 & 0.209 & 0.650 \\
0.6834 & 0.155 & 0.201 & 0.889 & 0.7867 & 0.222 & 0.203 & 0.673 \\
0.6878 & 0.170 & 0.190 & 0.871 & 0.7910 & 0.212 & 0.221 & 0.657 \\
0.6940 & 0.189 & 0.176 & 0.834 & 0.7939 & 0.209 & 0.216 & 0.692 \\
0.6985 & 0.181 & 0.199 & 0.817 & 0.7960 & 0.201 & 0.218 & 0.709 \\
0.7027 & 0.198 & 0.180 & 0.800 & 0.7980 & 0.197 & 0.217 & 0.715 \\
0.7070 & 0.205 & 0.184 & 0.792 & 0.8016 & 0.173 & 0.227 & 0.752 \\
0.7148 & 0.215 & 0.186 & 0.763 & 0.8036 & 0.174 & 0.221 & 0.775 \\
0.7190 & 0.210 & 0.206 & 0.738 & 0.8066 & 0.128 & 0.246 & 0.805 \\
0.7226 & 0.217 & 0.209 & 0.721 & 0.8091 & 0.121 & 0.243 & 0.856 \\
0.7276 & 0.223 & 0.204 & 0.717 & 0.8117 & 0.118 & 0.244 & 0.890 \\
0.7311 & 0.235 & 0.191 & 0.719 & 0.8154 & 0.113 & 0.235 & 0.922 \\
0.7350 & 0.231 & 0.208 & 0.694 & 0.8177 & 0.117 & 0.237 & 0.919 \\
0.7477 & 0.242 & 0.202 & 0.681 & 0.8210 & 0.112 & 0.243 & 0.918 \\
0.7515 & 0.240 & 0.208 & 0.669 & 0.8242 & 0.118 & 0.244 & 0.902 \\
0.7558 & 0.248 & 0.208 & 0.646 & & & & \\
\hline
\end{tabular}

HJD $=$ time +2446645.0 .

be determined only for $\mathrm{F}$ type (Nissen 1988). Mean values and the standard deviations were determined (Table 10) for the considered photometric data (KMJ and the present paper's) between these phase limits and, within these uncertainties, the magnitude and colors are practically the same. With these values a metallicity of -0.093 and 0.764 respectively, was calculated. Emphasis should be made on the fact that photometry in the present paper was carried out simultaneously in all the filters and that the dispersion is lower.
In view of the apparently large discrepancy, the determination of the metal content $[\mathrm{Fe} / \mathrm{H}]$ of $\mathrm{BP}$ Peg was also done considering the relations 4.6 .8 and 4.6 .9 provided by Rodríguez (1989). From these, the metal content for each set of data was $0.459 \pm 0.372$ for the photometry in present paper and $-0.400 \pm 0.738$ for the KMJ values for the 4.6 .8 equation and, analogously, of $0.359 \pm 0.275$ and $-0.235 \pm 0.451$ for the 4.6 .9 relation which when calculated from the standard deviations in the considered range phases, give the same values of metallicity within the uncertainties. 
Table 6. Unreddened photometry of DY Peg

\begin{tabular}{lccccccc}
\hline Time & $(b-y)_{0}$ & $m_{10}$ & $c_{10}$ & Time & $(b-y)_{0}$ & $m_{10}$ & $c_{10}$ \\
\hline 0.7113 & 0.195 & 0.132 & 0.785 & 0.7794 & 0.198 & 0.138 & 0.766 \\
0.7152 & 0.177 & 0.143 & 0.803 & 0.7806 & 0.195 & 0.124 & 0.804 \\
0.7203 & 0.140 & 0.152 & 0.896 & 0.7839 & 0.195 & 0.124 & 0.805 \\
0.7251 & 0.107 & 0.148 & 1.012 & 0.7850 & 0.184 & 0.139 & 0.778 \\
0.7304 & 0.091 & 0.156 & 1.064 & 0.7887 & 0.156 & 0.160 & 0.800 \\
0.7356 & 0.102 & 0.155 & 1.050 & 0.7925 & 0.139 & 0.160 & 0.865 \\
0.7409 & 0.122 & 0.161 & 0.982 & 0.7937 & 0.122 & 0.159 & 0.916 \\
0.7459 & 0.140 & 0.156 & 0.941 & 0.7994 & 0.088 & 0.168 & 1.002 \\
0.7510 & 0.162 & 0.143 & 0.893 & 0.8004 & 0.088 & 0.169 & 1.002 \\
0.7565 & 0.183 & 0.124 & 0.879 & 0.8014 & 0.078 & 0.174 & 1.021 \\
0.7618 & 0.185 & 0.133 & 0.830 & 0.8024 & 0.072 & 0.182 & 1.022 \\
0.7716 & 0.185 & 0.145 & 0.794 & 0.8034 & 0.073 & 0.166 & 1.059 \\
0.7731 & 0.196 & 0.139 & 0.788 & 0.8074 & 0.074 & 0.173 & 1.069 \\
0.7759 & 0.196 & 0.128 & 0.804 & 0.8085 & 0.074 & 0.181 & 1.054 \\
0.7769 & 0.194 & 0.129 & 0.804 & & & & \\
\hline
\end{tabular}

HJD $=$ time +2448925.0 .

Table 7. Unreddened photometry of YZ Boo

\begin{tabular}{lccc}
\hline Time & $(b-y)_{0}$ & $m_{10}$ & $c_{10}$ \\
\hline 0.8421 & 0.099 & 0.143 & 0.761 \\
0.8635 & 0.018 & 0.164 & 0.913 \\
0.8705 & 0.010 & 0.163 & 0.944 \\
0.8844 & 0.034 & 0.161 & 0.927 \\
0.8904 & 0.044 & 0.156 & 0.929 \\
0.8954 & 0.058 & 0.148 & 0.899 \\
0.9080 & 0.074 & 0.154 & 0.846 \\
0.9134 & 0.082 & 0.161 & 0.802 \\
0.9186 & 0.081 & 0.167 & 0.785 \\
0.9240 & 0.083 & 0.160 & 0.795 \\
0.9289 & 0.083 & 0.167 & 0.774 \\
0.9352 & 0.087 & 0.168 & 0.760 \\
0.9407 & 0.084 & 0.167 & 0.756 \\
0.9460 & 0.096 & 0.152 & 0.758 \\
0.9520 & 0.084 & 0.161 & 0.771 \\
0.9573 & 0.066 & 0.177 & 0.757 \\
0.9636 & 0.035 & 0.174 & 0.855 \\
\hline HJD = time +2449427.0. &
\end{tabular}

Table 8. Variations in $T_{\mathrm{e}}$ and $\log g$ during the pulsation cycle for the large amplitude stars

\begin{tabular}{lcccc}
\hline & $\log g_{\max }$ & $\log g_{\min }$ & $T_{\mathrm{e} \max }(\mathrm{K})$ & $T_{\mathrm{e} \min }(\mathrm{K})$ \\
\hline BP Peg & 4.144 & 3.441 & 8000 & 6860 \\
CY Aqr & 4.119 & 3.476 & 8320 & 6680 \\
DY Her & 4.264 & 3.610 & 8180 & 6800 \\
DY Peg & 3.960 & 3.324 & 8270 & 6910 \\
YZ Boo & 3.989 & 3.621 & 8065 & 7230 \\
\hline
\end{tabular}

From the determined values for the pulsational constant (Breger et al. 1996), as well as from their position in the period -luminosity-color diagrams (López de Coca et al. 1990), it has been determined that BP Peg, DY Her and YZ Boo are pulsating in the fundamental mode whereas CY Aqr and DY Peg do so in higher overtones ( $2 \mathrm{H}$ from the former method and between $2 \mathrm{H}$ and $3 \mathrm{H}$, from the last one). The derived mass values are 1.85, 2.1, $1.7,0.21$ and $0.225 M_{\odot}$, respectively.

What is immediately evident when comparing the physical parameters obtained from $u v b y-\beta$ photometric data with those reported in the literature, is the significant spread of values of the physical characteristics. The principal sources have been listed at the bottom of Table 9 . The comparison of the pulsational and evolutionary theories are in excellent agreement if the results derived by each method are examined. From these methods it was found that BP Peg, DY Her and YZ Boo are classical $\delta$ Scuti stars and fit the determinations of other authors, except for the metallicity found for YZ Boo. On the other hand, CY Aqr and DY Peg appear to be SX Phe stars of an older population than the $\delta$ Scuti stars. The scenario for these stars would be that they are in a more advanced evolutive stage which could correspond to prewhite dwarfs as the lower absolute magnitude indicates. No significant changes in the position of these stars in the HR diagram are expected because no significant changes have been found from the previously determined physical characteristics.

Acknowledgements. We would like to thank the staff of the OAN for their assistance in securing the observations. This work was partially supported by Conacyt through grants 3925E, E130-3059 and a previous collaboration program with CSIC which allowed the visit of R. Garrido to the OAN in 1986. Special recognition for his support in securing the 
Table 9. Comparison with the previously reported data in the literature

\begin{tabular}{|c|c|c|c|c|c|c|}
\hline ID & Source & {$[\mathrm{Fe} / \mathrm{H}]$} & $\log T_{\mathrm{e}}$ & $\log g$ & $T_{\mathrm{e}}(\mathrm{K})$ & $Q$ \\
\hline \multirow[t]{4}{*}{ BP Peg } & $\mathrm{PP}$ & 0.76 & 3.871 & 3.92 & 7430 & 0.045 \\
\hline & GKA & -0.08 & 3.875 & 3.85 & 7500 & \\
\hline & $\mathrm{KMcN} \& \mathrm{~J}$ & -0.08 & 3.873 & 3.85 & 7470 & \\
\hline & $\mathrm{McN}$ & -0.02 & & 3.87 & 7550 & 0.033 \\
\hline \multirow[t]{7}{*}{ CY Aqr } & $\mathrm{PP}$ & -0.90 & 3.875 & 3.91 & 7500 & 0.020 \\
\hline & GKA & -0.6 & 3.899 & 4.13 & 7925 & \\
\hline & $\mathrm{MB}$ & - & & 4.11 & & \\
\hline & $\mathrm{ER}$ & -0.7 & 3.880 & 3.85 & 7590 & 0.019 \\
\hline & $\mathrm{McN} \& \mathrm{~F}$ & -0.6 & - & 4.13 & 7930 & 0.033 \\
\hline & PLC & - & 3.893 & 3.95 & 7816 & 0.024 \\
\hline & $\mathrm{McN}$ & -1.40 & & 4.04 & 7720 & 0.031 \\
\hline \multirow[t]{8}{*}{ DY Her } & $\mathrm{PP}$ & 0.58 & 3.874 & 4.05 & 7490 & 0.061 \\
\hline & GKA & 0.30 & 3.853 & 3.66 & 7128 & - \\
\hline & $\mathrm{BC} \& \mathrm{R}$ & - & - & 3.87 & 7360 & - \\
\hline & $\mathrm{ER}$ & 0.30 & 3.865 & 3.72 & 7320 & 0.039 \\
\hline & $\mathrm{McN} \& \mathrm{~F}$ & 0.30 & 3.866 & 3.66 & 7130 & 0.033 \\
\hline & PLC & - & 3.860 & 3.59 & 7244 & 0.032 \\
\hline & $\mathrm{EA}$ & 0.16 & - & 3.87 & 7360 & - \\
\hline & $\mathrm{McN}$ & 0.00 & & 3.66 & 7250 & 0.033 \\
\hline \multirow[t]{7}{*}{ DY Peg } & $\mathrm{PP}$ & -0.56 & 3.880 & 3.75 & 7590 & 0.019 \\
\hline & GKA & -0.70 & 3.892 & 4.00 & 7800 & - \\
\hline & $\mathrm{MB}$ & - & - & 4.00 & - & - \\
\hline & $\mathrm{ER}$ & -0.6 & 3.885 & 3.89 & 7680 & 0.023 \\
\hline & $\mathrm{McN} \& \mathrm{~F}$ & -0.7 & 3.892 & 4.00 & 7800 & 0.033 \\
\hline & $K \& R$ & & & 4.04 & 7775 & 0.032 \\
\hline & $\mathrm{McN}$ & -0.65 & & 3.99 & 7705 & 0.032 \\
\hline \multirow[t]{8}{*}{ YZ Boo } & $\mathrm{PP}$ & -0.39 & 3.884 & 3.84 & 7650 & 0.032 \\
\hline & GKA & -0.10 & 3.884 & 3.97 & - & - \\
\hline & $\mathrm{MB}$ & - & - & 3.97 & - & - \\
\hline & $\mathrm{McN} \& \mathrm{~F}$ & -0.1 & 3.884 & 3.97 & 7650 & 0.033 \\
\hline & KJ & -0.25 & - & 3.71 & 7410 & \\
\hline & PLC & - & 3.880 & 3.80 & 7585 & 0.032 \\
\hline & $\mathrm{J} \& \mathrm{McN}$ & -0.025 & - & 3.95 & 7590 & 0.033 \\
\hline & $\mathrm{McN}$ & -0.60 & & 3.86 & 7490 & 0.032 \\
\hline
\end{tabular}

PP Present paper McN \& F McNamara \& Feltz (1978) GKA Andreasen (1983) KJ Kim \& Joner (1994b) MB Breger (1980) J \& McN Joner \& McNamara (1983) ER Rodríguez (1989) BC \& R Breger et al. (1978) EA Antonello (1990)

PLC López de Coca (1986) K \& R Kilambi \& Rahman (1993) KMcN \& J Kim et al. (1989) McN McNamara (1997).

observations of DY Her is acknowledged. Typing and proofreading were done by J. Orta and J. Miller, respectively. C. Guzman assisted us in the computing. This is part of the work for obtaining the Masters Degree of DG. We would like to acknowledge both fuitful discussions with Carlos Chavarría and suggestions of the referee which improved this paper.

\section{References}

Andreasen G.K., 1983, A\&A 121, 250

Antonello E., 1990, A\&A 230, 127

Arellano Ferro A., Parrao L., 1989, Reporte Técnico No. 57. Instituto de Astronomía, UNAM
Table 10. Mean values and standard deviations for BP Peg between phases 0.3 and 0.75

\begin{tabular}{llllll}
\hline & $\mathrm{V}$ & $b-y$ & $m_{1}$ & $c_{1}$ & $\mathrm{H} \beta$ \\
\hline $\mathrm{KMJ}$ & 12.059 & 0.256 & 0.148 & 0.795 & 2.736 \\
$\sigma$ & 0.058 & 0.033 & 0.041 & 0.051 & 0.047 \\
$\mathrm{PP}$ & 12.100 & 0.250 & 0.202 & 0.695 & 2.731 \\
$\sigma$ & 0.050 & 0.013 & 0.025 & 0.034 & 0.023 \\
\hline
\end{tabular}

Breger M., 1979, PASP 91, 5

Breger M., 1980, ApJ 235, 153

Breger M., Handler G., Garrido R., et al., 1996, Delta Scuti Star Newslett. 10, 24 
Breger M., 1998, ASPCS 135, 460

Breger M., Campos A.J., Roby S.W., 1978, PASP 90, 754

Broglia P., 1959, P. Contr. Obs. Astron. Milano-Merate, 142

Coates D.W., Fernely J.A., Sekiguchi K., Barnes T.G., Frueh M.L., 1994, MNRAS 266, 1

Crawford D.L., 1975, AJ 80, 955

Crawford D.L., 1979, AJ 84, 1858

Crawford D.L., Barnes J.V., 1970, AJ 75, 978

Crawford D.L., Mander J., 1966, AJ 71, 114

Dawei Y., Shiyang J., Quinquan T., Hongsheng W., 1993, IBVS 3831

Detre L., Chang Y.C., 1960, Acta Astr. Sin. 8, 50

Dziembowski W., Kozlowski M., 1974, Acta Astron. 24, 245

Elst E.W., 1972, A\&A 17, 148

Fitch W.S., 1973, A\&A 27, 171

Geyer E.H., Hoffmannn M., 1974, IBVS 936

Geyer E.H., Hoffmannn M., 1975, A\&A 21, 177

Gronbech B., Olsen E.H., 1977, A\&AS 27, 443

Hardie R.H., Tolbert C.R., 1961, ApJ 134, 581

Jiang S.-Y., 1986, Chinese A\&A 10, 115

Joner M.D., McNamara D.H., 1983, PASP 95, 433

Kilambi G.C., Rahman A., 1993, Bull. Astr. Soc. India 21, 573

Kim C., Joner M.D., 1994a, ApSS 218, 113

Kim C., Joner M.D., 1994b, ApSS 218, 137

Kim C., McNamara D.H., Joner M.D., 1989, PASP 101, 1009 KMS

López de Coca P., 1986, Tesis Doctoral. Universidad de Granada, Spain

López de Coca P., Rolland A., Rodríguez E., Garrido R., 1990, A\&AS 83, 51

Mahdy H.A., Szeidl B., 1980, Comm. Konkoly Obs. 74

McNamara D.H., 1978, PASP 90, 759

McNamara D.H., 1997, PASP 109, 1221

McNamara D.H., Feltz Jr. K.A., 1978, PASP 90, 275
Milone E.F., Wilson W.J.F., Fry D.J.I., 1994, PASP 106, 1120

Nissen P.E., 1984, Reporte Técnico, IA-UNAM, Mexico

Nissen P.E., 1988, A\&A 199, 146

Olsen E.H., 1983, A\&A 54, 54

Parrao L., Schuster W., Peña J.H., 1998 (in preparation)

Peniche R., González S.F., Peña J.H., 1985, PASP 97, 1172

Peniche R., Peña J.H., Díaz-Martínez S., Gómez T., 1990, RMxAA 20, 127

Peña J.H., Peniche R., 1986, A\&A 166, 211

Peña J.H., Peniche R., González S.F., Hobart M.A., 1987, RMxAA 14, 429

Peña J.H., Peniche R., Mujica R., et al., 1993, RMxAA 25, 129

Percy J.R., 1975, A\&A 43, 469

Powell J.M., Joner M.D., McNamara D.H., 1995, PASP 107, 225

Quigley R., Africano J., 1979, PASP 91, 230

Quigley R., Africano J., 1981, IBVS 2017

Relyea L.J., Kurucz R.L., 1978, ApJS 37, 45

Rodríguez E., 1989, Tesis Doctoral, Fotometría uvby $-\beta$ de estrellas variables tipo SX Phe y Delta Sct de gran amplitud. Universidad de Granada

Rodríguez E., Rolland A., López de Coca P., 1990, ApSS 169, 113

Rodríguez E., Rolland A., López de Coca P., 1993, A\&ASS 100,571

Rodríguez E., Rolland A., López de Coca P., García-Lobo E., Sedano J.L., 1992, A\&ASS 93, 189

Rolland A., Peña J.H., López de Coca P., Peniche R., González S.F., 1986, A\&A 168, 125

Szeidl B., 1979, IBVS 1718

Szeidl B., Mahdy H.A., 1981, Comm Konkoly Obs. 75, 1

Yang D., Jiang S., Tang Q., Wang H., 1993, IBVS 3831 